\title{
Dor persistente, anormalidades sensitivas, lesão de nervo e perda do implante após cirurgia com implantes dentais: sugestão de abordagem clínica*
}

\section{Persistent pain, sensory abnormalities, nervous injury and loss of implant after dental implant surgery: clinical approach suggestion}

José Tadeu Tesseroli de Siqueiraํㅗ Silvia Regina Dowgan Tesseroli de Siqueira².

*Recebido da Equipe de Dor Orofacial, Divisões de Odontologia e de Neurologia do Instituto Central do Hospital das Clínicas da Faculdade de Medicina da Universidade de São Paulo (FMUSP), São Paulo, SP.

\section{RESUMO}

JUSTIFICATIVA E OBJETIVOS: Alterações de sensibilidade trigeminal podem levar à dor neuropática após manipulação cirúrgica. Essas queixas geram dúvidas. Primeiro: são reais? Pois, nem sempre há relação causal entre implante-nervo. Segundo: o implante deve ser removido? O objetivo deste estudo foi rever a literatura sobre $\mathrm{o}$ assunto.

CONTEÚDO: A literatura é farta em informações sobre perdas de implantes relacionadas à osteointegração, mas são raros os estudos sobre perdas relacionadas à lesão de nervo ou à dor neuropática. O risco para longevidade de implantes é multifatorial. Contribuem: saúde do paciente, técnica cirúrgica, região receptora e ativação funcional, entre outros fatores. Dor é complicação com significativas implicações clínicas, psicológicas, sociais e legais; exigindo atenção especial aos pacientes, muitas vezes prolongada ou permanente. Atualmente a literatura especializada já é volumosa sobre prevalência de anormalidades sensitivas e lesão de nervo após procedimentos odontoló-

1. Cirurgião Dentista; Coordenador da Equipe de Dor Orofacial, Divisões de Odontologia e Neurologia, do Hospital das Clínicas da Faculdade de Medicina da Universidade de São Paulo. São Paulo, SP, Brasil.

2. Cirurgiã Dentista; Professora Doutora da Escola de Artes, Ciência e Humanidades da Universidade de São Paulo (USP). São Paulo, SP, Brasil.

\section{Endereço para correspondência:}

Dr. José Tadeu Tesseroli de Siqueira

Rua Maria Cândida, 135 - Vila Guilherme

02071-010 São Paulo, SP.

E-mail: jtts@uol.com.br gicos. Em conjunto, essas informações sugerem conduta que inclua exame minucioso, interconsultas e adoção de medidas preventivas, curativas ou paliativas para o tratamento da dor. A remoção do implante depende dessa análise, portanto, nem sempre é uma decisão simples.

CONCLUSÃO: Informações sobre remoção de implantes dentais devido à dor ou lesão de nervo são escassas nem sempre homogêneas, porém já alertam de que essa decisão nem sempre é simples. Ela depende da adoção de medidas diagnósticas, algumas especializadas, incluindo os exames de imagem. Diagnóstico e tratamento precoce da lesão de nervo e da dor neuropática são benéficos ao paciente e têm importantes implicações éticas e legais.

Descritores: Dor neuropática, Dor orofacial, Dor pós-operatória, Osteointegração.

\section{SUMMARY}

BACKGROUND AND OBJECTIVES: Trigeminal sensitivity changes may lead to neuropathic pain after surgical handling. These complaints generate questions. First: are they real? Because a causal relationship between implant and nerve is not always present. Second: should the implant be removed? This study aimed at reviewing the literature on the subject.

CONTENTS: The literature is prodigal on information about loss of implants related to osteointegration, but there are few studies on losses related to nerve loss or to neuropathic pain. The risk for implants longevity is multifactorial. Contributors are: patients' health, surgical technique, receptor region and functional activation, among other factors. Pain is a complication with significant clinical, psychological, social and legal implications, requi- 
ring special attention to patients, very often prolonged or permanent. Currently, specialized literature is already voluminous about the prevalence of sensory abnormalities and nervous injury after dental procedures. Together, these pieces of information suggest an approach including detailed exams, interconsultations and the adoption of preventive, curative or palliative measures to treat pain. Implant removal depends on this analysis and it is not always a simple decision.

CONCLUSION: Information about dental implants removal due to pain or nervous injury is scarce and not always homogeneous, however it already calls the attention to the fact that such decision is not always simple. It depends on the adoption of diagnostic measures, some of them specialized, including image exams. Early diagnosis and treatment of nervous injury and neuropathic pain are beneficial to patients and have major ethic and legal implications.

Keywords: Neuropathic pain, Orofacial pain, Osteointegration, Postoperative pain.

\section{INTRODUÇÃO}

O número de cirurgias odontológicas aumentou consideravelmente nos últimos anos no Brasil. Procedimentos cirúrgicos, como remoção do dente do siso, cirurgias ortognáticas e cirurgias ósseas para inserção de implantes dentários são geralmente seguros e com baixa frequência de complicações ou morbidade. Os implantes dentários, que apresentaram um significativo avanço para a reabilitação de pacientes com déficits morfológicos, funcionais ou estéticos, têm altas taxas de sobrevivência em longo prazo, e baixa frequência de complicações. Embora a maioria seja temporária, em alguns casos, como nas lesões de nervo e na dor persistente, podem ser prolongadas ou permanentes e o cirurgião deve estar preparado para enfrentá-las.

Por outro lado, uma das principais preocupações em implantodontia, tanto do profissional como do paciente, diz respeito à possibilidade de perda do implante, ou da não osteointegração. As perdas de implantes podem ocorrer durante o período de osteointegração, portanto, antes da sua ativação protética, ou em diferentes períodos de tempo após sua osteointegração primária e ativação funcional através da prótese.

Quando são adotados protocolos padronizados, como o clássico protocolo de Branemark e col. ${ }^{1}$, a ocorrência de falhas no período de osteointegração primária é motivo frequente de publicações científicas e, mesmo havendo variações, os percentuais de perdas são considerados baixos, as complicações temporárias e as causas parecem es- tar relacionadas fundamentalmente à indicação e à técnica cirúrgica $^{1,2}$. No Brasil, essa situação também se repete ${ }^{3,4}$. Com o aumento dos estudos sobre esse assunto e o aprimoramento para o controle dessas variáveis, o percentual de perdas de implantes é bem reduzido atualmente, desde que sejam adotadas medidas padronizadas para controle de indicações, da seleção do paciente e do procedimento cirúrgico, ainda que as causas destas perdas ainda não estejam completamente esclarecidas ${ }^{5}$. Tanto fatores relacionados à cirurgia ${ }^{1,2}$, como à condição médica do paciente ${ }^{6}$ devem ser considerados.

Outra causa possível de remoção do implante, embora incomum, talvez rara, e que nem sempre é levada em conta pelos profissionais da implantodontia, é a queixa de anormalidades sensitivas ou de dor persistente na área que recebeu o implante ${ }^{7,8}$, sendo que essa queixa pode estar associada ou não a alterações neuropáticas (parestesia, disestesia ou anestesia) ${ }^{9-12}$ e o seu tratamento pode ser complexo $^{13}$. O grande problema nesses casos, é que a dor não é prontamente reconhecida ou mesmo chega a ser negada pelo profissional. Esse comportamento tem implicações profundas, tanto na condição psicológica do paciente, como na relação paciente-cirurgião dentista. Também tem importantes implicações legais, médico-odontológicas, que não deveriam ser subestimadas.

Portanto, foi objetivo deste estudo fazer uma revisão sobre perdas de implantes dentários, especialmente quando relacionadas a anormalidades sensitivas trigeminais, lesão de nervo ou dor persistente, com a abordagem dos seguintes tópicos:

- Revisão sobre perdas de implantes devido a causas habitualmente relatadas na literatura científica;

- Revisão sobre perdas de implantes relacionadas à lesão de nervo, anormalidades sensitivas ou dor persistente em procedimentos de implantodontia;

- Sugestão de protocolo com tópicos que deveriam ser investigados durante a avaliação clínica.

\section{PERDAS DE IMPLANTES APÓS A OSTEOINTE- GRAÇÃO PRIMÁRIA}

Estudo retrospectivo publicado recentemente avaliou pacientes acompanhados por cerca de seis anos e identificou características diferentes, dependendo do tempo em que os implantes permaneceram nos pacientes. As perdas de implantes em período considerado de curto prazo foram mais comuns em mulheres jovens, em muitos casos eram apoios de coroas simples e a perda óssea na maioria deles foi leve. Já as perdas tardias dos implantes ocorreram mais em homens, nas regiões posteriores, e em geral não 
foram individuais, pois ocorreram em grupos; além disso, o comprometimento ósseo variou de moderado a grave ${ }^{14}$. Por outro lado, é importante salientar que a perda de implantes é maior em pacientes que apresentam condições de risco para osteointegração (fumantes com periodontite controlada), mesmo quando submetidos a um programa de controle permanente, em acompanhamento longitudinal de 10 anos $^{15}$.

Existem vários fatores de risco para a perda de implantes, como: região de inserção, topografia do implante, ativação funcional, e mesmo a comparação entre perdas de implantes com perdas das próteses, que merecem uma breve revisão (Tabela 1).

\section{Perda de implantes inseridos na maxila posterior}

Estudo de revisão sistemática em implantes inseridos imediata ou mediatamente em seios maxilares que foram elevados cirurgicamente, com ou sem enxertos autógenos, não conseguiu avaliar diferença nas perdas devido aos diversos fatores considerados de risco, como: fumantes, altura do rebordo ósseo residual, tipo do implante ou de superfície na região, uso de plasma-rico-em-plaquetas ou enxerto autógeno; por outro lado, a presença de barreira para fechamento lateral da parede sinusal foi considerado fator relevante para o sucesso do procedimento $^{16}$.

Tabela 1 - Causas habituais de perdas de implantes dentais osteointegrados, de acordo com estudos clínicos controlados e revisões sistemáticas.

\begin{tabular}{|c|c|c|}
\hline Fatores Estudados & Índice de Perdas / Fator de Risco (OR) & Autores \\
\hline Protocolo de Branemark e col. & Taxa de perda $=0,7 \%$. & Bornstein e col. $^{5}$ \\
\hline $\begin{array}{l}\text { Topografia: } \\
\text { Rugosa versus usinada } \\
\text { Todas as áreas }\end{array}$ & $\begin{array}{l}\text { Fumante: } \mathrm{OR}=3,1 \\
\text { Não fumante: } \mathrm{OR}=0,8\end{array}$ & Balshe e col. ${ }^{20}$ \\
\hline $\begin{array}{l}\text { Superfície rugosa } \\
\text { Carga imediata versus mediata } \\
\text { Seis meses de acompanhamento }\end{array}$ & $\begin{array}{l}\text { Taxa de Perda: } \\
\text { Implantes Imediatos }=11 \% \\
\text { Implantes Convencionais }=2 \%\end{array}$ & Ferreira e col. ${ }^{22}$ \\
\hline $\begin{array}{l}\text { Longevidade: curto e médio ou longo prazo } \\
\text { Peri-implantite } \\
\text { Complicações } \\
\text { Tipo de prótese }\end{array}$ & $\begin{array}{l}\text { } \text { Curto prazo: coroas unitárias. } \\
\text { Causa da perda: } 73,2 \% \text { por perda da osteointegração. } \\
\lambda \\
\text { Médio/Longo prazo: região posterior; perdas múltiplas; } \\
\text { perda óssea alveolar. } \\
\text { Causas das perdas: peri-implantite }(32 \%) \text {; sobrecarga } \\
(46,4 \%) \text {; fratura do implante }(6,2 \%) ; \text { ASA }>1 \text {. }\end{array}$ & Manor e col. ${ }^{14}$ \\
\hline $\begin{array}{l}\text { Levantamento do seio maxilar } \\
\text { Revisão sistemática ( } 3 \text { anos) }\end{array}$ & Taxa de perda $=16 \%$ & Pjetursson e col. ${ }^{17}$ \\
\hline Levantamento de seio maxilar & $\begin{array}{l}\text { Taxa de Perda }=3 \% \text { a } 16 \% . \\
\text { Fumante: } \mathrm{OR}=6,4 . \\
\text { Complicação cirúrgica: } \mathrm{OR}=8,2 .\end{array}$ & Huynh-Ba e col..$^{19}$ \\
\hline Implantes curtos em mandíbulas atróficas & Taxa de sobrevivência: $88 \%$ a $100 \%$. & Stellingsma e col. ${ }^{24}$ \\
\hline $\begin{array}{l}\text { Peri-implantite } \\
\text { Metanálise de estudos aleatórios } \\
\text { Usinado versus rugoso }\end{array}$ & Inconclusivo, parece não haver diferenças. & Esposito e col. ${ }^{23}$ \\
\hline $\begin{array}{l}\text { Osteointegração primária com } \\
\text { Implante cilíndrico com superfície rugosa / } \\
\text { Multicêntrico. } \\
\text { Todas as áreas. }\end{array}$ & $\begin{array}{l}\text { Taxa de perda }=0 \% \text { a } 18 \% \text {, dependendo } \\
\text { da experiência cirúrgica. } \\
\text { Taxa de perda }=4 \% \text {. }\end{array}$ & Siqueira e col. ${ }^{3}$ \\
\hline $\begin{array}{l}\text { Osteointegração primária com implantes cilíndri- } \\
\text { cos ou tipo parafuso com superfície rugosa. } \\
\text { Alunos de curso de especialização }\end{array}$ & Implantes $=3,7 \%$ & Baldo e col. ${ }^{4}$ \\
\hline $\begin{array}{l}\text { Perdas: Implantes versus Próteses com cinco anos } \\
\text { de acompanhamento. }\end{array}$ & $\begin{array}{l}\text { Próteses }=14,6 \% \\
\text { Próteses sobre implantes }=10,3 \% .\end{array}$ & Chung e col. ${ }^{26}$ \\
\hline $\begin{array}{l}\text { Perdas de próteses sobre implantes com seis } \\
\text { anos de acompanhamento. }\end{array}$ & $\begin{array}{l}\text { Próteses sobre implantes e dentes }=12,5 \% \text {. } \\
\text { Próteses unitárias }=14,6 \% \text {. } \\
\text { Próteses cimentadas }=6,8 \% \text {. } \\
\text { Próteses parafusadas }=16,6 \% .\end{array}$ & Weber e Sukotjo ${ }^{27}$ \\
\hline
\end{tabular}


Embora a perda de implantes em cirurgias de elevação do seio maxilar possa ser superior a de implantes inseridos em outras áreas (16,6\% em três anos), esse tipo de procedimento é considerado previsível e com poucas complicações cirúrgicas ${ }^{17}$. Além disso, este último estudo também mostrou que as perdas foram menores com implantes de superfícies rugosas e que a colocação de barreira na parede lateral do seio maxilar também foi outro aspecto considerado relevante.

Implantes inseridos em área previamente enxertada em maxila posterior tiveram taxa de sobrevivência de $86,1 \%$ contra a taxa de $96 \%$ para implantes inseridos na mesma região, porém não enxertada ${ }^{18}$.

Outro estudo de coorte em implantes inseridos na região posterior da maxila mostrou que a cirurgia para elevação do seio maxilar não foi considerada um fator de risco para a perda de implantes; entretanto, em pacientes fumantes $(\mathrm{OR}=6,4)$ e nos casos de complicações cirúrgicas $(\mathrm{OR}=$ $8,2)$ o risco de perda do implante em curto e médio prazo foi acentuado ${ }^{19}$. Já outro estudo semelhante, porém considerando todas as áreas maxilares, mostrou que implantes com superfície rugosa não aumentaram o risco para perdas de implantes em fumantes $(\mathrm{OR}=0,8)$, ao contrário dos implantes com superfície lisa $(\mathrm{OR}=3,1)^{20}$.

\section{Perda de implantes com função ou carga imediata}

Estudo recente mostrou que a taxa de sobrevivência em dois anos de acompanhamento para implantes com carga imediata foi de $99,4 \%$ na região mandíbula anterior e $97 \%$ na região posterior ${ }^{21}$. Outro estudo mostrou resultados sobre implantes inseridos em todas as regiões da boca e mostra o índice de perda em curto prazo (11\%), superior ao dos implantes com carga tardia $(2 \%)$, independente do tamanho dos implantes, embora fatores como o uso de fumo e saúde geral não tenham sido avaliados ${ }^{22}$. Neste estudo, a média de tamanho dos implantes utilizados no grupo de função imediata foi maior estatisticamente do que a média dos implantes usados para função mediata ( $\mathrm{p}$ $<0,001$ ), fato sugestivo de que o tamanho dos implantes nem sempre parece ser o fator determinante para a osteointegração, e tampouco para a sobrevida em curto e longo prazo dos implantes. A altura mínima dos implantes usados foi de $11 \mathrm{~mm}$.

\section{Influência da topografia de superfície e do tamanho do implante}

Embora os implantes em forma de parafuso sejam amplamente documentados ${ }^{2}$, os cilíndricos também têm ótima documentação. A influência da superfície, usinada versus rugosa, em complicações como a peri-implantite ainda não está bem definida nos estudos de revisão sistemática atuais, sendo necessários estudos com amostras mais homogêneas e por tempo mais prolongado para melhor análise comparativa ${ }^{23}$.

O comprimento dos implantes é praticamente padronizado pelo estudo de Branemark e col. ${ }^{1}$; entretanto o uso de implantes curtos pode ser uma alternativa em condições especiais como extremidades livres. Nesses casos a taxa de sobrevivência varia de $88 \%$ a $100 \%{ }^{24}$. A taxa de sobrevivência com esses implantes para 10 anos chegou a $92,7 \%{ }^{25}$.

\section{Perda de implantes comparativamente à perda das próteses}

Outro fator a ser considerado pelos profissionais ao indicarem reabilitação sobre implantes aos seus pacientes diz respeito à importância da conservação da prótese instalada sobre os implantes. A comparação entre falhas entre implantes e próteses sobre implantes, em um período de cinco anos de controle, mostrou que as perdas de implantes $(3,7 \%)$ foram inferiores às das próteses sobre implantes $(14,6 \%)^{26}$. Estudo sobre diferentes tipos de próteses sobre implantes observou variações na perda das próteses, desde 10,3\% em próteses retidas por implantes, $12,5 \%$ para próteses fixas retidas em implantes e dentes e 14,6\% para próteses unitárias sobre implantes, embora sem diferença estatisticamente significativa em seis anos de acompanhamento ${ }^{27}$. Neste mesmo estudo a taxa de perdas para próteses cimentadas foi de $6,8 \%$, sendo estatisticamente diferente da taxa de perdas para próteses parafusadas, que foi de $16,6 \%$. Este estudo mostrou que ainda não parece ser possível estabelecer um guia de orientações sobre a indicação de próteses sobre implantes.

\section{PERDAS DE IMPLANTES RELACIONADAS À LESÃO DE NERVO, ANORMALIDADE SENSITI- VA TRIGEMINAL OU DOR PERSISTENTE}

Até o momento praticamente não existem estudos sobre a prevalência da perda de implantes devido à dor persistente após procedimentos implantodônticos, entretanto essa é uma dúvida frequente do dentista quando ocorre lesão de nervo ou anormalidades sensitivas, com ou sem dor ${ }^{11,12}$. Por outro lado, a literatura científica sobre anormalidades sensitivas e dor persistente após procedimentos cirúrgicos em todas as regiões corpóreas mostra que 5\% dos pacientes com lesão neuropática desenvolvem dor crôni$\mathrm{ca}^{28}$ e que essa frequência se repete também após cirurgia ortognática $^{29}$. A tabela 2 mostra uma relação de estudos sobre a prevalência de anormalidades sensitivas trigemi- 
Tabela 2 - Anormalidades sensitivas, lesão de nervo ou dor persistente após procedimentos de implantodontia e a eventual associação com remoção do implante, de acordo com relatos de casos e estudos clínicos.

\begin{tabular}{|c|c|c|}
\hline Fatores Estudados & Índice de Perdas / Fator risco (OR) & Autores \\
\hline $\begin{array}{l}\text { Dor persistente após procedimentos de im- } \\
\text { plantodontia oral. }\end{array}$ & $\begin{array}{l}\text { Relatos de casos. Em um relato o implante na } \\
\text { maxila foi substituído duas vezes por outro } \\
\text { semelhante. }\end{array}$ & Siqueira e col. 1996; 2010. \\
\hline Lesão do NAI após cirurgias com implantes. & $\begin{array}{l}\text { Tratamento precoce com Laser terapêutico reduz } \\
\text { complicações. } \\
\text { Nada consta sobre a perda de implantes. }\end{array}$ & Ladalardo e col. 2004. \\
\hline $\begin{array}{l}\text { Lesão do NAI e do nervo lingual (NL) após } \\
\text { cirurgias de terceiros molares inferiores. }\end{array}$ & $\begin{array}{l}78 \% \text { dos cirurgiões relataram algum tipo de lesão } \\
\text { permanente do NAI; } 46 \% \text { do NL. } \\
\text { Não tem dados específicos sobre cirurgias com } \\
\text { implantes. }\end{array}$ & Robert e col. 2005. \\
\hline $\begin{array}{l}\text { Anormalidades sensitivas após cirurgia em } \\
\text { rebordo inferior anterior. }\end{array}$ & $\begin{array}{l}\text { Complicações sensitivas - 33\% dos pacientes. } \\
\text { Nada consta sobre a perda de implantes. }\end{array}$ & Abarca e col. 2006. \\
\hline $\begin{array}{l}\text { Dor persistente após cirurgia de implantes } \\
\text { Anormalidades sensitivas, lesão do NAI. } \\
\text { Estudo retrospectivo de } 10 \text { anos. }\end{array}$ & $\begin{array}{l}\text { O percentual de risco para lesões neurais após } \\
\text { procedimentos com implantes é de } 0,008 \% \text {, } \\
\text { sendo } 0,006 \% \text { persistentes. } \\
\text { Nada consta sobre a perda de implantes. }\end{array}$ & Libersa e col. 2007. \\
\hline $\begin{array}{l}\text { Lesão do NAI e lingual após cirurgias odon- } \\
\text { tológicas. } \\
\text { Estudo retrospectivo com } 73 \text { pacientes. }\end{array}$ & $\begin{array}{l}\text { Lesão do NAI após cirurgia com implantes den- } \\
\text { tais ocorreu em } 11,9 \% \text { da amostra. } \\
\text { Dor neuropática ocorreu em } 14,9 \% \text { de todos os } \\
\text { pacientes com lesão do NAI. } \\
\text { Nada consta sobre a perda de implantes. }\end{array}$ & Tay e Zuniga, 2007. \\
\hline $\begin{array}{l}\text { Lesão do NAI. } \\
\text { Estudo prospectivo não aleatório de } 52 \text { casos. }\end{array}$ & $\begin{array}{l}\text { Lesão após cirurgias de implantes ocorreu em } \\
10 \% \text { dos casos. } \\
\text { As lesões mecânicas, como na remoção de den- } \\
\text { tes do siso têm melhor prognóstico. } \\
\text { Quando há recuperação, esta ocorre geralmente } \\
\text { nos primeiros seis meses. } \\
\text { Nada consta sobre a perda de implantes. }\end{array}$ & Hillerup 2008. \\
\hline $\begin{array}{l}\text { Remoção do implante após lesão do NAI } \\
\text { Estudo tipo coorte com quatro casos. }\end{array}$ & A remoção precoce parece favorecer a evolução. & Khawaja e Renton 2009. \\
\hline $\begin{array}{l}\text { Lesão do NAI. } \\
\text { Estudo retrospectivo sobre complicações em } \\
\text { cirurgias de implantes dentais. }\end{array}$ & $\begin{array}{l}\text { Complicações = 13,35\%; Lesão do NAI = 2\%. } \\
\text { Nada consta sobre a perda de implantes. }\end{array}$ & Nóia e col. 2010. \\
\hline
\end{tabular}

$\mathrm{NAI}=$ nervo alveolar inferior

nais, lesão de nervo e dor persistente após procedimentos em implantodontia e sua associação com a remoção de implantes.

Embora esse tipo de queixa cause angústia e incompreensão, existem diversos estudos na literatura científica, como o que estima que mais de $70 \%$ dos dentistas já se defrontaram com queixas de anormalidades de sensibilidade, como parestesia/disestesia ou anestesia após pro- cedimentos odontológicos ${ }^{30}$. As causas mais comuns são os procedimentos anestésicos, endodônticos e cirúrgicos (p.ex.: anestesia do nervo alveolar inferior, remoção do dente do siso, cirurgia de implantes, cirurgias ortognáticas e cirurgias de tumores) $)^{31-35}$.

O desconhecimento sobre esse tipo de problema acaba confundindo-o com aqueles relacionados à dor pós-operatória inflamatória, gerando dúvidas sobre o implante 
e sua relação com os ramos trigeminais, principalmente com o nervo alveolar inferior (NAI). A situação se complica quando a queixa relaciona-se a implantes inseridos na maxila.

Estudo sobre a frequência de anormalidades sensitivas após a colocação de implantes com função imediata (Branemark Novum) em área considerada segura, como o rebordo inferior anterior, mostrou que $33 \%$ dos pacientes (n $=19)$ relataram tais complicações ${ }^{36}$. Testes psicológicos não mostraram diferenças destes pacientes com outros submetidos ao mesmo tratamento, porém sem complicações sensitivas. Entretanto, alterações de sensibilidade mecânica leve (alodínia) no lábio inferior foram mais frequentes nos pacientes com complicações. A duração desse desconforto foi inferior a três meses em 11 dos 19 pacientes e as regiões afetadas foram o lábio inferior, a gengiva ou o mento. Em cinco pacientes foram afetadas as funções da fala e ingestão de líquidos (insensibilidade ou babação). A queixa mais comum em nove pacientes foi de parestesia do lábio; um paciente não faria novamente a cirurgia se soubesse dessa possibilidade.

Estudo francês sobre queixas ao seguro saúde, que analisou um período de 10 anos sobre pacientes que reclamavam de alterações sensitivas após procedimentos odontológicos, mostrou que essas anormalidades ocorreram após procedimentos endodônticos e cirúrgicos (remoção do terceiro molar e colocação de implantes) $)^{32}$. A seguir a percentagem de risco para esses problemas:

a) Para os procedimentos endodônticos foi de $0,086 \%$ para problemas transitórios e $0,013 \%$ para os persistentes; 2. Para procedimentos cirúrgicos foi de $0,15 \%$, sendo de $0,029 \%$ para problemas persistentes (principalmente remoção do terceiro molar);

3. Para procedimentos implantodônticos o risco foi de $0,008 \%$, sendo de $0,006 \%$ para lesões persistentes.

No total os autores observaram que no período estudado, entre 517 dentistas, o risco anual para uma anormalidade sensitiva trigeminal foi de $0,196 \%$, e que a possibilidade de um dentista causar essa alteração permanentemente foi de 1:2,04 dentistas. Esse estudo não considerou a especialidade do dentista.

No caso de lesão de nervo durante cirurgias bucais (remoção de terceiros molares), de acordo com pesquisa que avaliou a resposta de 535 cirurgiões da Califórnia ${ }^{37}$, houve a seguinte informação, decorrente de um período de 12 meses: 94,5\% cirurgiões relataram algum tipo de lesão do NAI e 53\% no nervo lingual (NL); 78\% relataram lesões permanentes do NAI e $46 \%$ do NL; foi estimada 4:1000 lesão do NAI por exodontias de terceiros molares, e 1:1.000 para o NL. Lesões permanentes do NAI ocorreram em 1:1.000 dentes removidos e do NL em 1:2.500 pacientes. As lesões foram relacionadas com a experiência clínica do profissional.

Um único estudo de coorte, com apenas quatro casos, avaliou o resultado da remoção precoce dos implantes quando a queixa do paciente decorria de lesão de nervo ${ }^{12}$. Observaram que pode haver reversão da sensibilidade quando os implantes forem removidos até seis dias depois da cirurgia inicial. O uso precoce de corticosteroides e de anti-inflamatórios não hormonais parece contribuir para recuperação mais rápida. Com base nos seus dados eles sugeriram um protocolo para avaliação padronizada dos pacientes que apresentem anormalidades sensitivas trigeminais após procedimentos implantológicos. Estudo experimental confirma a eficácia da dexametasona na prevenção da dor neuropática, embora pareça não ter efeito quando esta última já está instalada ${ }^{38}$. Outro estudo, utilizando laser terapêutico mostrou também que o atendimento precoce da lesão do NAI, após cirurgia de implantes dentais, é benéfico ao paciente, reduzindo as complicações de longo prazo, como a parestesia e a dor neuropática ${ }^{10}$. Do mesmo modo, quando a dor neuropática está presente, seu tratamento precoce é altamente benéfi$\mathrm{co}^{13}$.

Outro estudo brasileiro ${ }^{39}$ mostrou que entre 532 pacientes submetidos à cirurgia com implantes osteointegráveis, 71 deles tiveram complicações, sendo 3,6\% $(n=19)$ no intraoperatório, das quais três foi lesão do NAI; 9,75\% $(\mathrm{n}=52)$ no pós-operatório, sendo que parestesia do NAI ocorreu em 15,37\% $(\mathrm{n}=8)$. Não foram relatados mais detalhes à respeito.

Existem relatos de dor persistente após implantodontia em que os implantes são removidos ou substituídos diversas vezes sem alteração da queixa, ou mesmo com sua piora. Isso ocorre devido à incompreensão de que essa dor difere da dor inflamatória habitual ${ }^{11}$. Quando a dor é neuropática, nem sempre ela é prontamente identificada, muitas vezes a queixa do paciente é até confrontada pelo profissional, fato que gera insegurança, angústia ou raiva no paciente e deteriora as relações entre paciente-dentista ${ }^{40}$. O reconhecimento precoce dessa condição permite que o paciente seja devidamente avaliado, orientado e tratado, reduzindo eventuais problemas ético-legais que o desconhecimento desse tipo de queixa acaba gerando.

\section{AVALIAÇÃO DE PACIENTES COM SUSPEITA DE COMPLICAÇÕES NEURAIS APÓS PROCEDI- MENTOS ODONTOLÓGICOS, INTRA OU EXTRA- ORAIS}

Os dados da literatura científica vigente, em conjunto com a experiência clínica e de pesquisa da Equipe de Dor 
Orofacial das Divisões de Odontologia e Neurologia do Hospital das Clínicas da Faculdade de Medicina da Universidade de São Paulo permitem a elaboração de um protocolo com tópicos que deveriam ser abordados durante a avaliação de pacientes com queixas indicativas de envolvimento neuropático na região do complexo nuclear trigeminal (Quadro 1).

Quadro 1 - Sugestões de tópicos que deveriam ser considerados durante a avaliação de queixas de anormalidades sensitivas ou dor neuropática após procedimentos odontológicos ou bucomaxilofaciais

\section{Lesão do Nervo Trigêmeo}

Procedimentos odontológicos com maior risco de complicações neuropáticas

Cirurgias

Exodontia do terceiro molar inferior

Cirurgia de implantes

Cirurgia ortognática

Cirurgia de tumores

\section{Endodontia}

Anestesia de tronco / ramo nervoso

Dentística (restaurações de resina composta) *

Tipo de complicação cirúrgica

Anormalidades sensitivas sem lesão de

tronco ou ramo nervoso

Lesão de tronco ou ramo nervoso

Sem dor

Com dor neuropática (cerca de 5\%

dos que têm lesão do nervo)

Tipo de lesão de tronco / ramo do nervo (adaptado ${ }^{44,45}$ )

Neuropraxia - bloqueio de condução;

sem degeneração

Axonotmesis - lesão moderada; possível

recuperação

Neurotmesis - lesão completa; possível

recuperação com cirurgia

Quanto à duração da anormalidade / lesão / dor

Temporária

Prolongada

Permanente

Queixas habituais

Parestesia

Mordida de lábios, bochecha, etc.

Sensação estranha no dente, implante,

boca ou face

Queimação, formigamento

Dor

* Raros relatos até o presente momento

\section{DISCUSSÃO}

Como observado na literatura consultada, felizmente as perdas de implantes são pequenas e não são impedimentos para seu uso. Entretanto, fica evidente que as complicações relacionadas à sua perda não são homogêneas e têm diferentes níveis de complexidade. Isso deve ser levado em conta para o esclarecimento correto do paciente e para evitar mal entendido quando esses problemas ocorrerem.

Um aspecto que chama atenção e que pode ser observado na tabela 2 são de que as informações científicas sobre perda em geral de implantes já são bem avançadas e baseiam-se em estudos clínicos controlados, revisões sistemáticas ou metanálises. Ao contrário, as informações sobre remoção de implantes associadas a queixas de lesão de nervo, anormalidades sensitivas ou dor persistente baseiam-se ainda muito em relatos de casos e estudos descritivos não controlados. Uma coorte, com apenas quatro casos, mostra a implicação da remoção do implante em relação à recuperação do nervo alveolar inferior em longo prazo ${ }^{12}$. Em geral, a literatura específica já alerta para significativa prevalência dessas anormalidades trigeminais, as quais merecem atenção do cirurgião, já no pré-operatório, inclusive para notificar o risco dessa complicação ao paciente ${ }^{29,34,35}$. O problema torna-se mais relevante ainda ao se observar a frequência de transtornos da saúde mental, como a depressão, em pacientes com dores crônicas ${ }^{40}$, que pode ocorrer nessas complicações cirúrgicas.

Em relação à presença de desconfortos ou de dor persistente, mesmo não havendo perda do implante, e mesmo ele estando osteointegrado, há uma dúvida constante do paciente e também do profissional, principalmente quando tem pouca experiência com pacientes com dor crônica. Então, sempre fica a dúvida se o implante deve ser removido ou não. Esse tipo de desconfiança ocorre em outras condições de dor crônica, quando não há diagnóstico da dor, e esta é persistente e refratária aos tratamentos, exatamente por ainda não ter sido identificada e não ter sido aplicado o tratamento adequado. Uma das situações mais comuns em que isso ocorre é a neuralgia trigeminal, cuja dor pode parecer uma dor de dente, o que leva à remoção de muitos dentes sadios, contribuindo mais ainda para aumentar o sofrimento do paciente e também o afetando emocionalmente ${ }^{41}$. Quando a queixa de dor está presente, ao contrário da maioria das complicações dos implantes, ela é acompanhada por transtornos afetivos, na área da saúde mental, como ansiedade e depressão, e isso complica ainda mais a avaliação clínica desses pacientes, pois dor é uma experiência multidimensional e certamente complexa. 
No caso do implante já osteointegrado, o diagnóstico que motiva essa queixa é mais relevante do que pensar na remoção do implante, principalmente quando os exames de imagens não mostram áreas de lesão óssea ao seu redor. Neste caso pode ser indispensável uma avaliação especializada, seja por dentista treinado nesse tipo de problema, seja por neurologista envolvido também com essa condição álgica. Quando a dor neuropática está presente, associada ou não à lesão evidente de nervo, a literatura mostra que o tratamento precoce é altamente benéfico ${ }^{13}$; e que existem opções terapêuticas próprias para o tratamento desse tipo complexo de dor.

Situações como essas exigem que o cirurgião identifique de imediato a existência da lesão de nervo, ou de alterações sensitivas a ela relacionadas, como a própria dor, e providenciam atenção imediata a essas queixas ou encaminhem o paciente para avaliação especializada. Além disso, embora os exames de imagem sejam importantes, nem sempre são suficientes para identificar a lesão do nervo ou a origem da dor persistente, crônica ou neuropática, pois nem sempre basta lesão intraóssea para justificar esse tipo de dor. Além disso, as lesões de nervo, independente da presença de dor, têm características próprias e a recuperação da estrutura lesada depende muito de como ela ocorre $^{42,43}$.

Particularmente nos casos de alterações sensitivas, como desconfortos, dormência ou dor, com ou sem lesão de nervo, há forte impacto na qualidade de vida do paciente, afetando atividades corriqueiras como mastigar ou ingerir líquidos. No caso de dor persistente há uma grande insatisfação, principalmente quando os pacientes não foram alertados sobre essa possibilidade antes da cirurgia. A complexidade desses casos, muitas vezes é tão grande, e com enormes implicações legais, que os cirurgiões deveriam ficar mais atentos, para evitar transtornos que deles possam advir, incluindo a deterioração na relação paciente-dentista.

Felizmente, o número de complicações após procedimentos de implantodontia é pequeno, mas não desprezível; entretanto, no caso de anormalidades sensitivas trigeminais, lesão de nervo e/ou dor neuropática pode ocorrer incapacitação para atividades da vida diária, insatisfação do paciente, pelo fato de ficar com uma sequela importante e que afeta sua qualidade de vida, além da necessidade de uso prolongado de fármacos ou mesmo tratamentos neurocirúrgicos.

Esses aspectos merecem mais estudos para a determinação de fatores associados que sejam de risco ou desencadeantes desse tipo de complicação. No momento atual, os profissionais devem estar atentos para a identificação de alterações de sensibilidade que indiquem um componente neuropático após a colocação de implantes, independente da presença de dor, uma vez que esta pode ser a evolução desses casos. É importante lembrar que quaisquer procedimentos cirúrgicos lesam terminações nervosas livres, por isso a necessidade do acompanhamento cuidadoso, melhorando o pós-operatório do paciente e prevenindo a cronificação da dor. Sendo assim, o fundamental é que haja a compreensão por parte do cirurgião de que essa queixa deve ser investigada e que seu mecanismo é diferente do da dor inflamatória convencional.

\section{CONCLUSÃO}

Informações sobre remoção de implantes dentais devido à dor ou lesão de nervo são escassas e nem sempre homogêneas, porém já alertam de que essa decisão nem sempre é simples. Ela depende da adoção de medidas diagnósticas, algumas especializadas, incluindo os exames de imagem. Diagnóstico e tratamento precoce da lesão de nervo e da dor neuropática são benéficos ao paciente e têm importantes implicações éticas e legais.

\section{AGRADECIMENTOS}

À Fundação de Apoio à Pesquisa do Estado de São Paulo (FAPESP), pelo suporte aos estudos sobre dor orofacial e sensibilidade trigeminal (2007/00934-2; 2007/04156-4) e ao Sistema de Próteses e Implantes INP pelo apoio à realização deste estudo.

\section{REFERÊNCIAS}

1. Branemark PI, Hansson BO, Adell R, et al. Osseointegrated implants in the treatment of the edentulous jaw. Experience from a 10-year period. Scand J Plast Reconstr Surg 1977;11(Suppl 16):1-132.

2. Adell R, Lekholm U, Rockler B, e col. A 15-year study of osseointegrated implants in the treatment of the edentulous jaw. Int J Oral Surg 1981;10(6):387-416.

3. Siqueira JTT, Dias PV, Salomão M, et al. Estudo multicêntrico de osteointegração com implantes cilíndricos, corpo com anéis e superfície rugosa por tratamento mecânico-químico. Fase I - Avaliação da osteointegração primária (ao término da fase de cicatrização). Rev ABO Nac 1977;5(3):164-70.

4. Baldo VO, Velasco Dias P, Salomão M, Siqueira JTT. Estudo clínico retrospectivo sobre a osteointegração primária em implantes de titânio com superfície rugosa por jateamento - Comparação entre cilindros e parafusos. BCI - Rev Brasil Cir Implant 2001;8(30):115-9.

5. Bornstein MM, Halbritter S, Harnisch H, et al. A re- 
trospective analysis of patients referred for implant placement to a specialty clinic: indications, surgical procedures, and early failures. Int J Oral Maxillofac Implants 2008;23(6):1109-16.

6. American Society of Anesthesiologists. New classification of physical status. Anesthesiology 1963;24:111.

7. Gregg JM. Neuropathic complications of mandibular implant surgery: review and case presentations. Ann R Australas Coll Dent Surg 2000;15:176-80.

8. Rodríguez-Lozano FJ, Sanchez-Pérez A, Moya-Villaescusa MJ, et al. Neuropathic orofacial pain after dental implant placement: review of the literature and case report. Oral Surg Oral Med Oral Pathol Oral Radiol Endod 2010;109(4):e8-12.

9. Siqueira JTT, Volpe A, Salomão M, et al. Cirurgia de implantes osteointegrados: a importância do diagnóstico diferencial no controle da dor pós-operatória. Considerações sobre dois casos clínicos. Rev Bras Implant 1996;11-14.

10. Ladalardo TCC, Brugnera JR A, Bologna ED, et al. Laserterapia no tratamento de déficit neurosensorial decorrente de procedimento cirúrgico em Implantodontia. Rev ImplantNews 2004;1(2):155-8.

11. Siqueira JTT, Siqueira SRDT. Dor orofacial pós-operatória persistente: o risco de iatrogenia. Relato de caso. Rev Dor 2010;11(2):180-4.

12. Khawaja N, Renton T. Case studies on implant removal influencing the resolution of inferior alveolar nerve injury. Br Dent J 2009;206(7):365-70.

13. Park JH, Lee SH, Kim ST. Pharmacologic management of trigeminal nerve injury pain after dental implant surgery. Int J Prosthodont 2010;23(4):342-6.

14. Manor Y, Oubaid S, Mardinger O, et al. Characteristics of early versus late implant failure: a retrospective study. J Oral Maxillofac Surg 2009;67(12):2649-52.

15. Matarasso S, Rasperini G, Iorio Siciliano V, et al. A 10-year retrospective analysis of marginal bone-level changes around implants in periodontally healthy and periodontally compromised tobacco smokers. Clin Oral Implants Res 2010;21(9):898-903.

16. Wallace SS, Froum SJ. Effect of maxillary sinus augmentation on the survival of endosseous dental implants. A systematic review. Ann Periodontol 2003;8(1):328-43. 17. Pjetursson BE, Tan WC, Zwahlen N, et al. A systematic review of the success of sinus floor elevation and survival of implants inserted in combination with sinus floor elevation. J Clin Periodontol 2008;35(8 Suppl):216-40.

18. Barone A, Orlando B, Tonelli P, et al. Survival rate for implants placed in the posterior maxilla with and without sinus augmentation: a comparative cohort study. J Periodontol 2011;82(2):219-26.

19. Huynh-Ba G, Friedberg JR, Vogiatzi D, et al. Implant failure predictors in the posterior maxilla: a retrospective study of 273 consecutive implants. J Periodontol
2008;79(12):2256-61.

20. Balshe AA, Eckert SE, Koka S, et al. The effects of smoking on the survival of smooth- and rough-surface dental implants. Int J Oral Maxillofac Implants 2008;23(6):1117-22.

21. Kacer CM, Dyer JD, Kraut RA. Immediate loading of dental implants in the anterior and posterior mandible: a retrospective study of 120 cases. J Oral Maxillofac Surg 2010;68(11):2861-7.

22. Ferreira LCP, Brito CR, Lehn CN, et al. Avaliação de implantes osteointegráveis submetidos à função imediata comparados à função tardia. Rev ImplantNews 2009;6(6):611-622.

23. Esposito M, Murray-Curtis L, Grusovin MG, et al. Interventions for replacing missing teeth: different types of dental implants. Cochrane Database Syst Rev 2007;17(4):CD003815.

24. Stellingsma K, Vissink A, Meijer HJ, et al. Implantology and the severely resorbed edentulous mandible. Crit Rev Oral Biol Med 2004;15(4):240-8.

25. Deporter D, Watson P, Pharoah M, et al. Ten-year results of a prospective study using porous-surfaced dental implants and a mandibular overdenture. Clin Implant Dent Relat Res 2002;4(4):183-9.

26. Chung WE, Rubenstein JE, Phillips KM, et al. Outcomes assessment of patients treated with osseointegrated dental implants at the university of Washington graduate Prosthodontic Program, 1988 to 2000. Int J Oral Maxillofac Implants 2009;24(5):927-35.

27. Weber HP, Sukotjo C. Does the type of implant prosthesis affect outcomes in the partially edentulous patient? Int J Oral Maxillofac Implants 2007;22(Suppl):140-72.

28. Kehlet H, Jensen TS, Woolf C. Persistent postsurgical pain: risk factors and preventions. Lancet 2006;367(9522):1618-25.

29. Jääskeläinen SK, Teerijoki-Oksa T, Virtanen A, et al. Sensory regeneration following intraoperatively verified trigeminal nerve injury. Neurology 2004;62(11):1951-7. 30. Misch CE, Resnik R. Mandibular nerve neurosensory impairment after dental implant surgery: management and protocol. Implant Dent 2010; 19(5):378-86.

31. Eliav E, Gracely RH. Sensory changes in the territory of the lingual and inferior alveolar nerves following lower third molar extraction. Pain 1998;77(2):191-9.

32. Libersa P, Savignat M, Tonnel A. Neurosensory disturbances of the inferior alveolar nerve: a retrospective study of complaints in a 10-year period. J Oral Maxillofac Surg 2007;65(8):1486-9.

33. Tay AB, Zuniga JR. Clinical characteristics of trigeminal nerve injury referrals to a university centre. Int $\mathrm{J}$ Oral Maxillofac Surg 2007;36(10):922-7.

34. Hillerup S. Iatrogenic injury to oral branches of the trigeminal nerve: records of 449 cases. Clin Oral Investig 2007;11(2):133-42.

35 . Hillerup S. Iatrogenic injury to the inferior alveolar 
nerve: etiology, signs and symptoms, and observations on recovery. Int J Oral Maxillofac Surg 2008;37(8):704-9. 36. Abarca M, van Steenberghe D, Malevez C, et al. Neurosensory disturbances after immediate loading of implants in the anterior mandible: an initial questionnaire approach followed by a psychophysical assessment. Clin Oral Invest 2006;10(4):269-77.

37. Robert RC, Bacchetti P, Pogrel MA. Frequency of trigeminal nerve injuries following third molar removal. J Oral Maxillofac Surg 2005;63(6):732-6.

38. Han SR, Yeo SP, Lee MK, et al. Early dexamethasone relieves trigeminal neuropathic pain. J Dent Res 2010;89(9):915-20.

39. Nóia CF, Ortega-Lopes R, Moraes M, et al. Complicações decorrentes do tratamento com implantes dentários: Análise retrospectiva de sete anos. Rev Assoc Paul
Cir Dent 2010;64(2):146-9.

40. McWilliams LA, Cox BJ, Enns MW. Mood and anxiety disorders associated with chronic pain: an examination in a nationally representative sample. Pain 2003;106(1-2): 77-83.

41. de Siqueira SR, Nóbrega JC, Valle LB, et al. Idiopathic trigeminal neuralgia: Clinical aspects and dental procedures. Oral Surg Oral Med Oral Pathol Oral Radiol Endod 2004;98(3):311-5.

42. Seddon JJ. Three types of nerve injury. Brain 1943;66:237-40.

43. Sunderland S. A classification of peripheral nerve injuries produced by a loss of function. Brain 1952;74:491-505.

Apresentado em 16 de dezembro de 2010.

Aceito para publicação em 02 de maio de 2011. 